\title{
Association of Care Practices with Suicide Attempts in US Veterans Prescribed Opioid Medications for Chronic Pain Management
}

\author{
Jinwoo J. Im, $P h D^{1,2,3}$, Ross D. Shachter, $P h D^{2}$, Elizabeth M. Oliva, PhD ${ }^{7}$, Patricia T. Henderson, $\mathrm{MS}^{7}$, \\ Meenah C. Paik, $M P H^{7}$, and Jodie A. Trafton, PhD ${ }^{1,4}$ for the PROGRES Team \\ ${ }^{1}$ Center for Health Care Evaluation, Department of Veterans Affairs, Menlo Park, CA, USA; ${ }^{2}$ Department of Management Science and Engineering, \\ Stanford University, Stanford, CA, USA; ${ }^{3}$ Management of Innovation Program, Daegu Gyeongbuk Institute of Science and Technology, Daegu, \\ South Korea; ${ }^{4}$ Department of Psychiatry and Behavioral Sciences and Center for Health Policy, Stanford University School of Medicine, Stanford, \\ CA, USA.
}

IMPORTANCE: Patients receiving opioid therapy are at elevated risk of attempting suicide. Guidelines recommend practices to mitigate risk, but it is not known whether these are effective.

OBJECTIVE: Our aim was to examine associations between the receipt of guideline-recommended care for opioid therapy and risk of suicide attempt.

DESIGN, SETTING, AND PARTICIPANTS: This was a retrospective analysis of administrative data for all Veteran patients prescribed any short-acting opioids on a chronic basis or any long-acting opioids from the Veterans Health Administration during fiscal year 2010.

MAIN OUTCOMES AND MEASURES: Multivariate, mixed-effects logistic regression analyses were conducted to define the associations between the risk of suicide attempt and receipt of guideline-recommended care at the individual level and rates of use of recommended care at the facility level, while accounting for patient risk factors. RESULTS: At the individual level, having a mood disorder was highly associated with suicide attempts (odds ratios [ORs] $=3.5,3.9 ; 95 \%$ confidence intervals [CIs]=3.3-3.9, 3.3-4.6 for chronic short-acting and long-acting groups, respectively). At the facility level, patients on opioid therapy within the facilities ordering more drug screens were associated with decreased risk of suicide attempt (ORs=0.2, 0.3; CIs=0.1-0.3, 0.2-0.6 for chronic shortacting and long-acting groups, respectively). In addition, patients on long-acting opioid therapy within the facilities providing more follow-up after new prescriptions were associated with decreased risk of suicide attempt $(\mathrm{OR}=0.2, \mathrm{CI}=0.0-0.7)$, and patients on long-acting opioid therapy within the facilities having higher sedative coprescription rates were associated with increased risk of suicide attempt (OR=20.3, $\mathrm{CI}=1.1-382.2)$.

CONCLUSIONS AND RELEVANCE: Encouraging facilities to make more consistent use of drug screening, provide follow-up within 4 weeks for patients initiating new opioid prescriptions, and avoid sedative co-prescription in combination with long-acting opioids may help prevent suicide attempts. Some clinicians may selectively employ guideline-recommended practices with at-risk patients.

KEY WORDS: opioid therapy; suicide attempt; clinical practice guideline.

Received March 11, 2014

Revised August 6, 2014

Accepted January 27, 2015

Published online February 19, 2015
J Gen Intern Med 30(7):979-91

DOI: $10.1007 / \mathrm{s} 11606-015-3220-y$

(C) Society of General Internal Medicine 2015

\section{INTRODUCTION}

Patients receiving opioid therapy (OT) are at elevated risk of attempting suicide, ${ }^{1}$ but definitive research to guide clinical practice for risk mitigation is lacking. Unmanaged severe, chronic and intermittent pain conditions increase risk of suicide attempt, ${ }^{2-9}$ a finding that might encourage providers to take aggressive action to reduce chronic pain in at-risk patients. However, available opioids also provide means for attempting suicide, ${ }^{10}$ potentially discouraging prescribing of these analgesics. Competing pressures have led experts to focus on clinical strategies for reducing the risk of adverse events in patients prescribed opioids.

In 2010, the Veterans Health Administration (VHA) and Department of Defense released a Clinical Practice Guideline for the Management of Opioid Therapy for Chronic Pain (CPG for OT). ${ }^{11}$ Like other guidelines for OT for chronic pain, ${ }^{12,13}$ this guideline provides recommendations, based primarily on expert consensus, for recognizing patient populations at higher risk of opioid-related adverse events, and intensifying care or monitoring such patients to minimize risk. For all patients, especially higher risk patients, the guideline recommends intensified clinical management, such as timely follow-up after new prescriptions.

To track and encourage adherence to this emerging practice standard, a team including the guideline co-chairs; VHA national pain, pharmacy, and addictions policy leadership; primary care, specialty pain and mental health clinicians; and measurement experts developed a set of administrative databased process measures to evaluate current use of key guideline-recommended practices. ${ }^{14}$ Examination of variation in use of recommended practices across VHA showed two common patterns: (1) for some recommendations, facility rates were consistent across the system, but indicated incomplete adherence to the recommendation; (2) for other recommendations, rates of adherence varied greatly across facilities. Because most guideline recommendations have been based on 
expert consensus rather than evidence-based research, it is not clear whether recommended care delivery, or the observed variations in clinical practice, impact patient risk for adverse outcomes.

Here, we examine associations between use of guideline recommendations for OT and risk of suicide attempt. Because clinical practices may be adapted based on patient characteristics associated with risk, solely examining patient-level or facility-level associations between clinical practice and outcomes can encourage logically flawed inferences regarding clinical practice effects. ${ }^{15}$ Thus, we aim to examine associations between individual-level and facility-level delivery of guidelinerecommended care and individual-level suicide attempts. Notably, although the guideline provides detailed information to guide dosing of specific opioids during initiation, titration, and cessation, it does not specify prescription details such as choice of drug. Thus, such variables, while potentially relevant for suicide risk, were not included in these analyses.

\section{METHODS}

\section{Data}

All Veteran patients prescribed any short-acting opioids on a chronic basis (chronic short-acting, CSA) or any long-acting (LA) opioids from the VHA during fiscal year 2010 (FY10) were included in this analysis (see Appendix 1 for CSA and LA definitions). Chronic use of short-acting opioids was defined as having at least 90 days of supply in FY10. ${ }^{14}$ Notably, $91 \%$ of the patients in the LA group also received short-acting opioids in FY10, but were exclusively counted in the LA group. Clinical expert advisors suggested that long-acting medications may pose greater risk for adverse events, and that these medications are typically prescribed by specialists and in non-primary care settings. Here, we examine populations receiving any long-acting and chronic short-acting opioids separately, to clarify if and where differences in the patient risk and care practices exist. All patients receiving long-acting prescriptions were included, rather than just those with more than 90 days of supply, to allow for comparison with other published data on this population. ${ }^{16}$ Given concerns about prescribing opioids to patients diagnosed with substance use disorder (SUD), additional analyses examined individuallevel and facility-level factors associated with suicide attempts among the patient population.

Data were extracted from the VHA National Patient Care Database and VHA Decision Support System pharmacy and laboratory files; databases were linked by matching patients' scrambled social security numbers. This study was approved and overseen by the Stanford University Human Research Protection Program and the VA Palo Alto Health Care System Research and Development Committee.

\section{Measures}

The dependent variable in this study is a binary indicator of whether a Veteran patient had completed or attempted suicide after being prescribed any CSA or LA opioids (hereafter referred to as "suicide attempts"). We included all documented incidents, confirmed or suspected, occurring in the first 180 days after any prescription was initiated during FY10 (see Appendix 2 for definition).

Individual-Level Predictors. Demographic variables included age, gender, and marital status. Age was categorized into three groups: younger than 31 years old, 31-55 years old, and greater than 55 years old. Physical/mental health status was represented by indicator variables for medical frailty, drug/alcohol/mood disorder, and traumatic brain injury (see Appendix 3 for definitions).

We used binary indicator variables to identify whether patients received guideline-recommended care. The covariates were chosen from the $C P G$ for $O T^{11}$ and employed metrics developed for VHA that measured delivery of guidelinerecommended care. ${ }^{14}$ Here, we include the following guideline-recommended care delivery measures (see Appendix 4 for rationale and definitions): psychosocial assessment or therapy, sedative co-prescription (e.g., benzodiazepines, barbiturates, and carisoprodol [Soma]), follow-up, drug screening, medication management, avoidance of sole reliance on opioids, and SUD specialty treatment.

Facility-Level Predictors. We included facility-level guideline-recommended care delivery rates in the analysis. These rates were calculated for each VHA facility by dividing the number of patients on OT receiving each guidelinerecommended service by the total number of patients on OT within the VHA facility.

\section{Statistical Analyses}

To examine individual-level and facility-level factors associated with patients' suicide attempts, we conducted multivariate mixed-effects logistic regression, with a random effect for each facility to account for the clustering of patients within VHA facilities. ${ }^{15}$ Mixed-effects logistic regression is a branch of logistic regression having a binary outcome as a dependent variable (e.g., whether a patient attempted suicide after being prescribed only CSA or LA opioids). Traditional single-level logistic regression assumes observations are independent conditional on the covariates and uncorrelated residual errors, which may not hold when analyzing nested data. ${ }^{16,17}$ In contrast, mixed-effects logistic regression considers variations derived from the data's hierarchical structure and allows for simultaneous examination of effects of facility-level and individual-level variables on individual-level outcomes while accounting for the non-independence of observations within facilities. ${ }^{17}$. 
The analyses were performed for CSA and LA OT groups separately, and in addition, for SUD-diagnosed patients within each group. We chose a $p$ value of $<$ 0.05 as a threshold for statistical significance. All multivariate mixed-effects logistic regression analyses were conducted using the glmmPQL function in the MASS pack$\mathrm{age}^{18}$ of $\mathrm{R}$ statistical software (version 2.15.0). ${ }^{19}$.

\section{RESULTS}

\section{Overview}

Table 1 shows individual-level predictors of suicide attempts among patients on OT. During FY10, 487,462 Veteran patients received OT at 139 VHA facilities. Of these patients, $393,657(80.8 \%)$ received CSA opioids, and 93,805(19.2\%) received LA opioids. Within the first 180 days after opioid prescriptions, 6,482(1.6\%) CSA patients and 1,982(2.1\%) LA patients attempted suicide. According to Table 2, 47,771(12.1\%) were diagnosed with SUD, and 3,440(7.2 \%) of them attempted suicide among patients on CSA OT. Among patients on LA OT, 13,732(14.6\%) were diagnosed with SUD, and $1,144(8.3 \%)$ of them attempted suicide.

Table 3 shows facility-level guideline-recommended care delivery rates for patients receiving OT. For patients on both CSA and LA OT, the guideline-recommended care most frequently provided was rehabilitation medicine (72.4 and $74.7 \%$ for patients on CSA and LA OT, respectively). The guideline-recommended care least frequently provided was specialty pain pharmacotherapy with TCAs (14.3 and $19.0 \%$ for patients on CSA and LA OT, respectively). The standard deviations of facility-level propensities to order drug screens for patients on both CSA and LA OT were largest compared to their mean propensities. This indicates that drug screens were not provided consistently to patients on CSA or LA OT across facilities.

\section{Chronic Short-Acting Opioid Prescribed Population}

Table 4 summarizes the multivariate, mixed-effects logistic regression results for patients receiving CSA OT. Insignificant variables are shaded in the table. At the individual level, suicide attempts were associated with being younger, female, currently unmarried, and having medical frailty, drug/alcohol/mood disorder, or traumatic brain injury. In particular, having a mood disorder was highly associated with suicide attempts (odds ratio $[\mathrm{OR}]=3.5,95 \%$ confidence interval $[\mathrm{CI}]=3.3-3.9$ ). Moreover, patients receiving psychosocial assessment/therapy, sedative co-prescription, drug screening, medication management, rehabilitation medicine, and anti-inflammatory or specialty pain pharmacotherapy were associated with increased risk of suicide attempt. At the facility-level, patients prescribed CSA OT within VHA facilities ordering more drug screens were associated with decreased risk of suicide attempt $(\mathrm{OR}=0.2, \mathrm{CI}=0.1-0.3)$.

Table 4 also summarizes results for SUD-diagnosed patients on CSA OT. In general, the pattern of individual-level findings were similar to those for the entire CSA OT group; however, unlike the entire CSA OT group, gender and antiinflammatory and specialty pain pharmacotherapy (SNRIs) were not significant predictors of suicide attempts for the SUD-diagnosed group on CSA OT. Similar to results for the entire CSA OT group, having a mood disorder was highly associated with suicide attempts $(\mathrm{OR}=5.4, \mathrm{CI}=4.6-6.3)$. At the facility-level, SUD-diagnosed patients on CSA OT within VHA facilities ordering regular drug screens (defined as at least one drug screen for every 90 days of opioid supply) more consistently or providing more SUD specialty treatment/SUDspecific pharmacotherapies were associated with decreased risk of suicide attempt $(\mathrm{ORs}=0.3, \mathrm{CI}=0.2-0.6)$.

\section{Long-Acting Opioid Prescribed Population}

Results for patients on LA OT (Table 5) were consistent with results for patients on CSA OT. For the entire LA OT group, suicide attempts were associated with being younger, currently unmarried, and having medical frailty, drug/alcohol $/ \mathrm{mood}$ disorder, or traumatic brain injury. Similarly, having a mood disorder was highly associated with suicide attempts $(\mathrm{OR}=3.9$, $\mathrm{CI}=3.3-4.6$ ). Patients receiving psychosocial assessment/therapy, sedative co-prescription, drug screening, medication management, rehabilitation medicine, or specialty pain pharmacotherapy were associated with increased risk of suicide attempt. Consistent with the entire CSA OT group, the LA OT group within VHA facilities ordering more drug screens was associated with decreased risk of suicide attempt $(\mathrm{OR}=0.3$, $\mathrm{CI}=0.2-0.6$ ); in addition, the LA OT group within VHA facilities providing more follow-up after new prescriptions was associated with decreased risk of suicide attempt $(\mathrm{OR}=0.2, \mathrm{CI}=0.0-0.7)$, and the LA OT group within VHA facilities having higher sedative co-prescription rates was associated with increased risk of suicide attempt $(\mathrm{OR}=20.3$, $\mathrm{CI}=1.1-382.2$ ).

Table 5 also summarizes results for the SUD-diagnosed group on LA OT. The pattern of individual-level findings was identical to those for the entire LA OT group, except that being currently unmarried was not associated with suicide attempts for patients with SUD. Similar to the entire LA OT group, among SUD-diagnosed group on LA OT, having a mood disorder was highly associated with suicide attempts ( $\mathrm{OR}=5.9, \mathrm{CI}=4.4-7.8)$. At the facility-level, SUD-diagnosed group on LA OT within VHA facilities providing more rehabilitation medicine were associated with decreased risk of suicide attempt (OR=0.3, CI=0.1-0.9), while SUDdiagnosed patients within VHA facilities having higher sedative co-prescription rates and providing more SNRIs were associated with increased risk of suicide attempt $(\mathrm{ORs}=30.2$ and 62.2; CIs $=1.1-829.3$ and 2.2-1,769.3, respectively). 
Table 1. Individual-Level Predictors and Suicide Attempts in the Entire Population Prescribed Opioid in the VHA in FY10

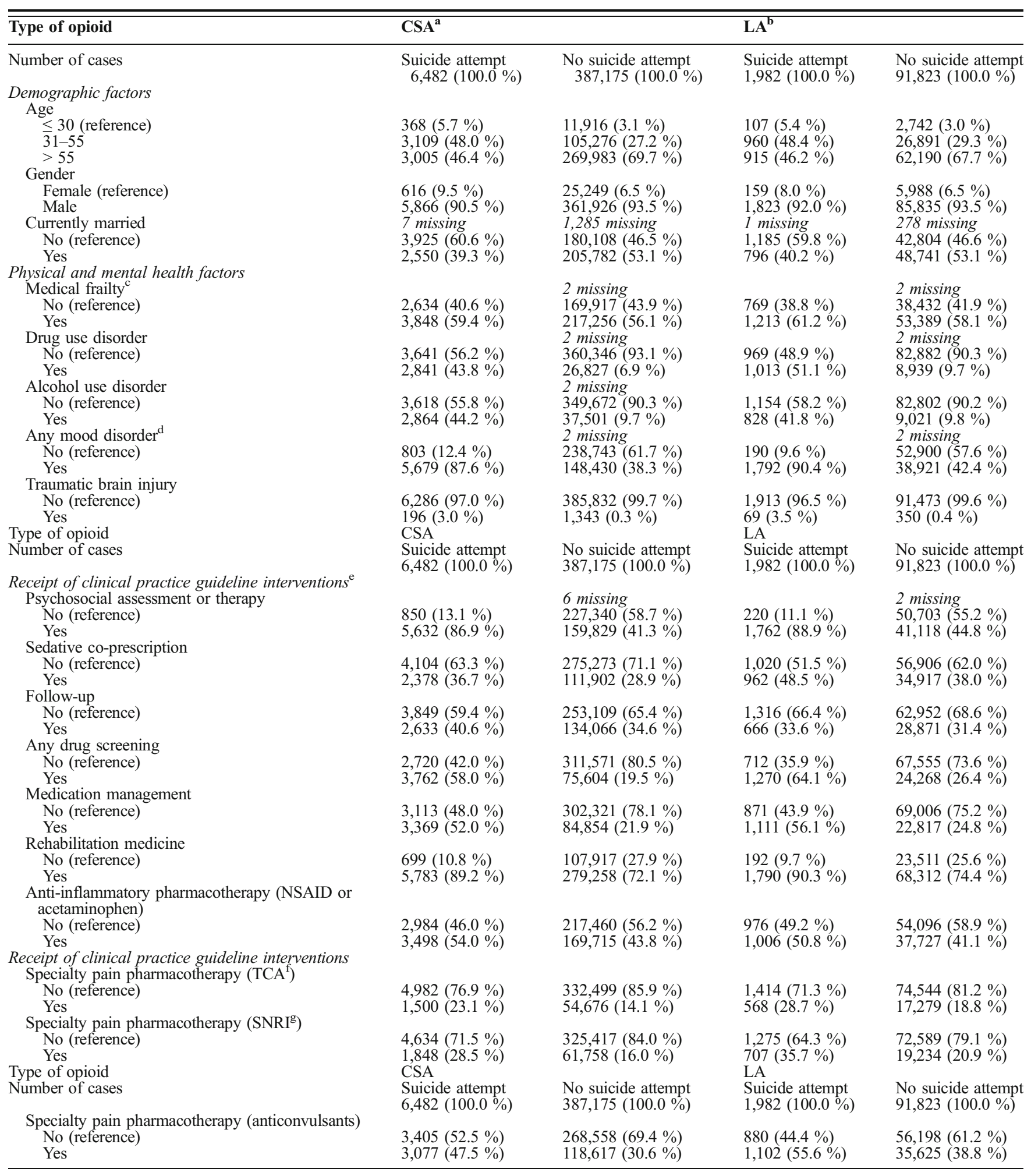

a Short-acting opioid medications on a chronic basis (chronic short-acting)

${ }^{b}$ Long-acting

${ }^{c}$ Congestive heart failure, cardiac arrhythmia, valvular disease, pulmonary circulation disorder, peripheral vascular disorder, paralysis, other neurological disorder, chronic pulmonary disease, hypothyroidism, renal failure, liver disease, peptic ulcer disease - excluding bleeding, aids, lymphoma, metastatic cancer, solid tumor without metastasis, rheumatoid arthritis/collagen vascular, coagulopathy, weight loss, fluid and electrolyte disorders, blood loss anemia, or deficiency anemias

${ }^{d}$ Depression, anxiety disorder, PTSD, or affective psychosis (bipolar)

e Please refer to Appendix Table 4

${ }^{f}$ Tricyclic antidepressants

${ }^{g}$ Serotonin-norepinephrine reuptake inhibitors 
Table 2. Individual-Level Predictors and Suicide Attempts in the Population Diagnosed with Substance Use Disorder and Prescribed Opioid in the VHA in FY10

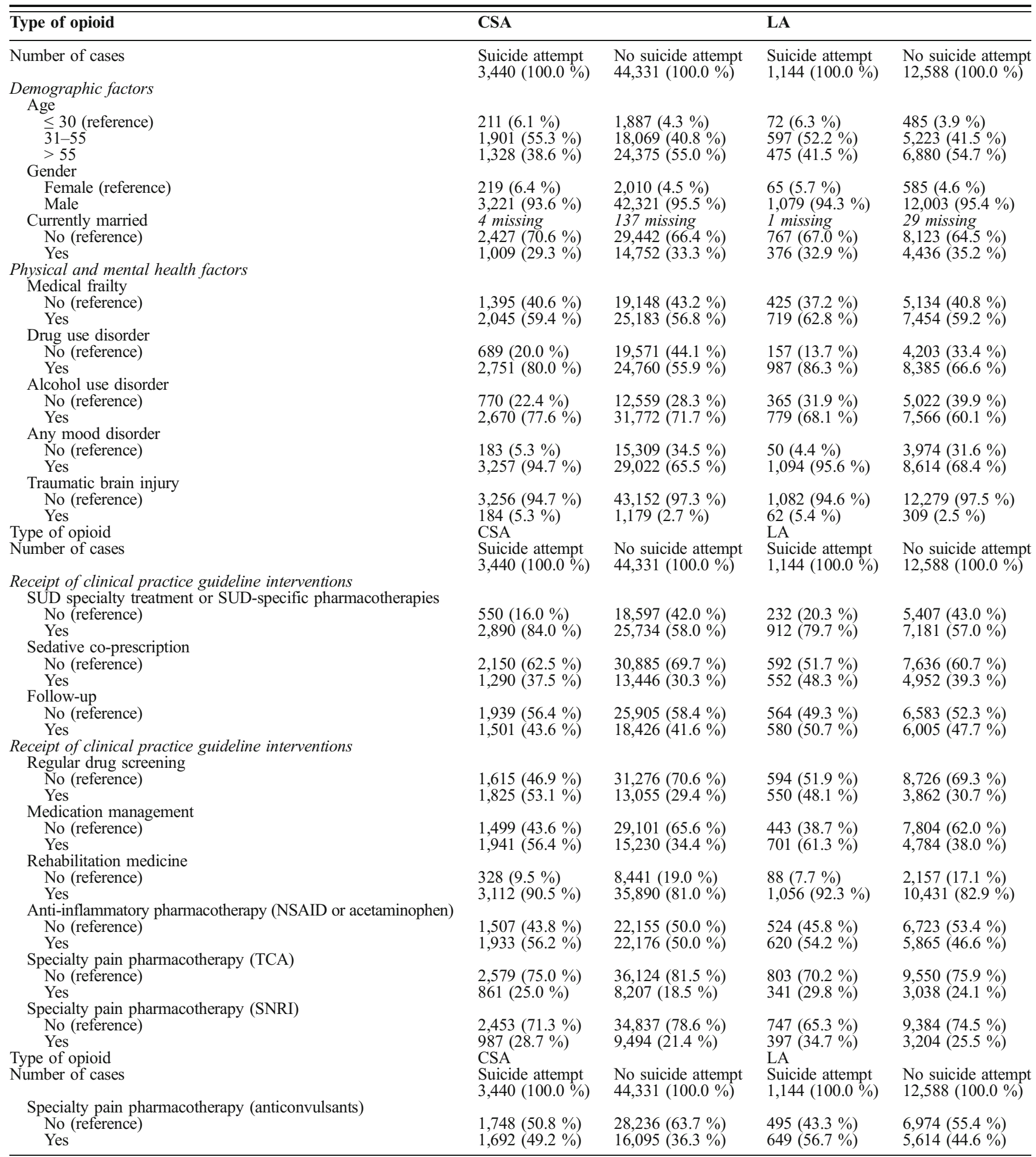

\section{DISCUSSION}

Consistent with expectations, patients with medical frailty, drug/alcohol/mood disorder, and/or traumatic brain injury evidenced higher risk of suicide attempt.
A striking finding was a pattern of conflicting individuallevel and facility-level associations with suicide attempts. We believe this pattern suggests that at the individual-level, guideline recommendations were applied to higher-risk patients selectively, as patient receipt of guideline-recommended care 
Table 3. Facility-Level Guideline Adherence Rates for the Opioid Prescribed Population in the VHA in FY10

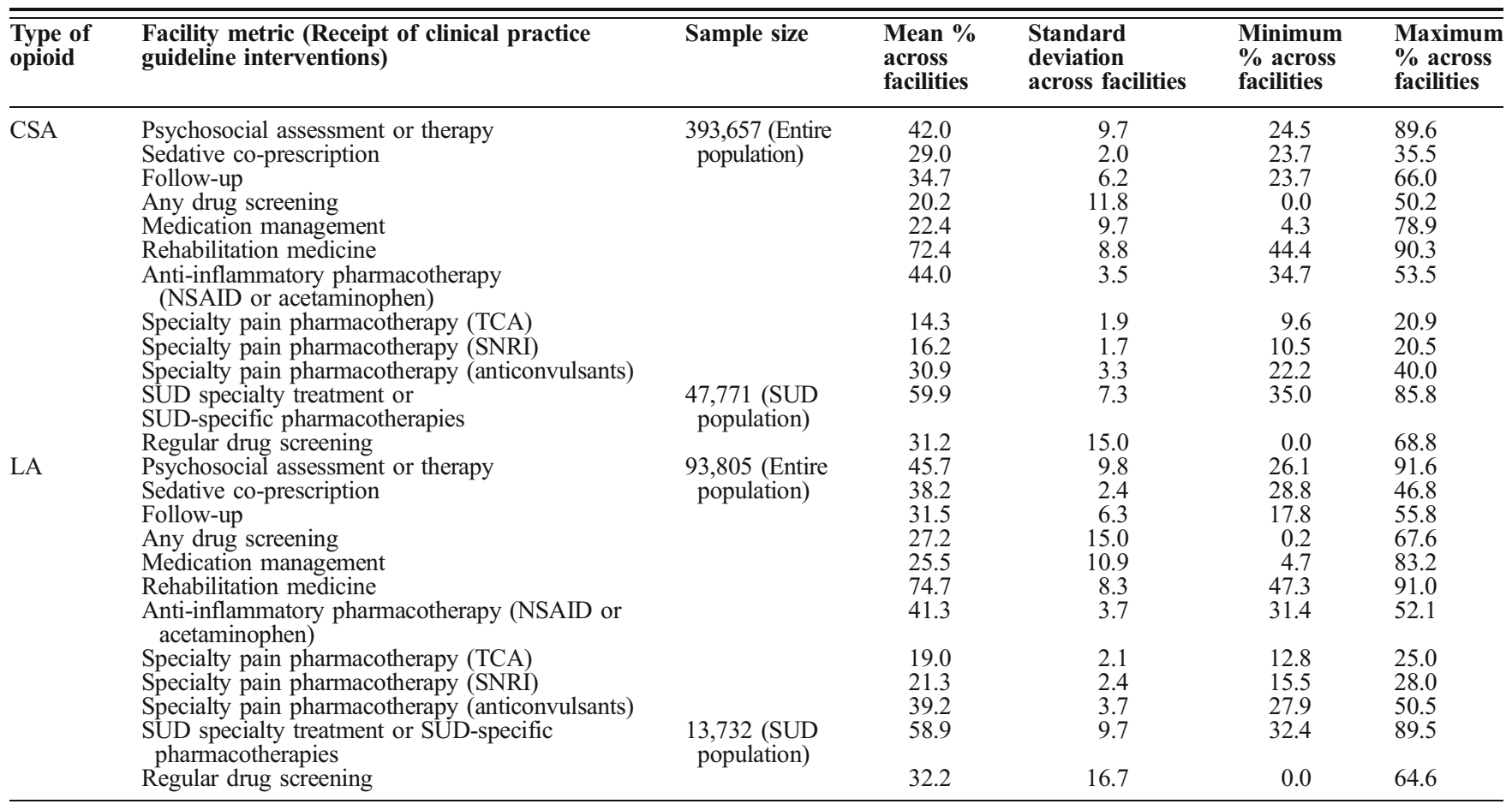

was associated with increased risk of suicide attempt across most measures. ${ }^{19}$ This suggests that, within VA, patients with increased risk of suicide attempt, most likely related to more severe or unmanaged chronic pain or comorbid mental health conditions, tend to receive more intensive, cautious, and comprehensive chronic pain and opioid management. We assume that this association is due to selective treatment of patients with greater pain or mental health severity or risk, rather than to guideline-recommended treatment increasing suicidality. An exception to this pattern was the recommendation to avoid co-prescribing sedative medications with opioids; patients on LA OT and co-prescribed sedatives were at higher risk of suicide attempts at both the individual and the facility level.

Examination of associations between facility-level guideline-recommended care delivery rates and risk of suicide attempt help to determine the value of regular or universal implementation of specific recommendations. Across CSA and LA populations, more consistent use of drug screening was associated with lower risk of suicide attempts. Drug screening is recommended to identify patients who may be using illicit substances or non-prescribed medications, so that the clinician can address risks and engage the patient in treatment for substance use problems. Active substance use is known to increase risk for suicide ${ }^{20}$; insofar as drug screening is a first step towards helping patients cease active substance use, it should reduce this risk. Moreover, identification of substance use should trigger clinicians to reduce a patient's access to abusable medications that could be used in a suicide attempt. Reducing access to means of suicide is a wellestablished method for reducing suicidal behaviors. ${ }^{21}$ If drug screening causally reduces suicide attempt risk, we estimate that universal drug screening would reduce the risk of suicide attempt in patients prescribed CSA or LA opioids by a factor of 3 to 6 across the models. These findings support the guideline recommendation that all patients considered for or receiving an opioid prescription should be screened for drug use.

Consistently providing follow-up visits to patients initiating new LA opioids was associated with lower risk of suicide attempts. Follow-up within 4 weeks after new opioid prescriptions may help identify patients for whom opioids worsen mental health or are not effective in reducing severe pain. For patients on LA opioids, if follow-up is causally related to risk, consistently completing follow-up encounters within the time frame recommended in the guideline is estimated to reduce the risk of suicide by roughly 5.4 times.

Sedative drugs and/or alcohol are often found in fatal opioid poisonings, both intentional and accidental, ${ }^{22,23}$ and sedative prescription has been associated with higher risk of fatal accidental drug poisoning ${ }^{24}$ and suicidal ideation and attempts. ${ }^{25}$ Here, in both the overall and SUD-diagnosed subpopulation prescribed LA opioids, higher facility-level rates of sedative co-prescription were associated with much higher (20-30 times) risk of suicide attempt. Studies in VA populations have suggested that patients on high-dose opioids and with mental health disorders are more likely to also be coprescribed sedatives. ${ }^{26,27}$ Acknowledging that it is possible that suicide attempts are more commonly observed in people co-prescribed opioids and benzodiazepines because these are 
Table 4. Multivariate, Mixed-Effects Logistic Regression Model Predicting Suicide Attempts of the CSA Opioid Prescribed Population in the VHA

\begin{tabular}{|c|c|c|c|c|c|c|c|}
\hline \multirow[t]{29}{*}{ Overall $^{\mathrm{h}}$} & (Intercept) & $\begin{array}{c}\text { Estimate } \\
-5.448\end{array}$ & OR & $\begin{array}{l}\text { SE } \\
0.814\end{array}$ & $\begin{array}{l}\text { DF } \\
392,202\end{array}$ & $\begin{array}{c}\mathrm{t} \text { value } \\
-6.692\end{array}$ & $\begin{array}{l}\mathrm{p} \text { value } \\
<0.001\end{array}$ \\
\hline & $\begin{array}{l}\text { Demographic factors } \\
\text { Age (reference: } \leq 30)\end{array}$ & -0.295 & 0.745 & 0.024 & \multirow{3}{*}{392,202} & -12.121 & \\
\hline & Gender (reference: female) & -0.123 & 0.885 & 0.048 & & -2.540 & 0.011 \\
\hline & Currently married (reference: no) & -0.133 & 0.875 & 0.029 & & -4.596 & $<0.001$ \\
\hline & $\begin{array}{l}\text { Physical and mental health factors } \\
\text { Medical frailty (reference: no) }\end{array}$ & 0.197 & 1.218 & 0.029 & \multirow{5}{*}{392,202} & 6.782 & $<0.001$ \\
\hline & Drug use disorder (reference: no) & 0.885 & 2.424 & 0.034 & & 26.145 & $<0.001$ \\
\hline & Alcohol use disorder (reference: no) & 0.690 & 1.994 & 0.033 & & 21.212 & $<0.001$ \\
\hline & Any mood disorder (reference: no) & 1.266 & 3.548 & 0.045 & & 28.330 & $<0.001$ \\
\hline & Traumatic brain injury (reference: no) & 0.269 & 1.309 & 0.087 & & 3.087 & 0.002 \\
\hline & Individual-level receipt of clinical practice guideline interventions & & & & \multirow{10}{*}{392,202} & & \\
\hline & Psychosocial assessment or therapy (reference: no) & 0.861 & 2.366 & 0.044 & & 19.431 & $<0.001$ \\
\hline & Sedative co-prescription (reference: no) & 0.109 & 1.115 & 0.029 & & 3.815 & $<0.001$ \\
\hline & Any drug screening (reference: no) & 0.983 & 2.673 & 0.033 & & 29.891 & $<0.001$ \\
\hline & Medication management (reference: no) & 0.443 & 1.557 & 0.030 & & 14.840 & $<0.001$ \\
\hline & Rehabilitation medicine (reference: no) & 0.305 & 1.357 & 0.045 & & 6.817 & $<0.001$ \\
\hline & $\begin{array}{l}\text { Anti-inflammatory pharmacotherapy (NSAID or acetaminophen) } \\
\text { (reference: no) }\end{array}$ & 0.067 & 1.069 & 0.028 & & 2.412 & 0.016 \\
\hline & Specialty pain pharmacotherapy (TCA) (reference: no) & 0.085 & 1.089 & 0.033 & & 2.579 & 0.010 \\
\hline & Specialty pain pharmacotherapy (SNRI) (reference: no) & 0.080 & 1.083 & 0.031 & & 2.586 & 0.010 \\
\hline & Specialty pain pharmacotherapy (anticonvulsants) (reference: no) & 0.223 & 1.250 & 0.028 & & 8.005 & $<0.001$ \\
\hline & $\begin{array}{l}\text { Facility-level receipt of clinical practice guideline interventions } \\
\% \text { Psychosocial assessment or therapy (reference: no) }\end{array}$ & -0.576 & 0.562 & 0.407 & \multirow{10}{*}{128} & -1.415 & 0.160 \\
\hline & $\%$ Sedative co-prescription (reference: no) & -0.195 & 0.823 & 1.833 & & -0.106 & 0.915 \\
\hline & \% Follow-up (reference: no) & -1.289 & 0.275 & 0.668 & & -1.931 & 0.056 \\
\hline & $\%$ Any drug screening (reference: no) & -1.782 & 0.168 & 0.347 & & -5.129 & $<0.001$ \\
\hline & $\%$ Medication management (reference: no) & 0.332 & 1.394 & 0.387 & & 0.857 & 0.393 \\
\hline & $\%$ Rehabilitation medicine (reference: no) & -0.487 & 0.615 & 0.472 & & -1.032 & 0.304 \\
\hline & $\begin{array}{l}\text { \% Anti-inflammatory pharmacotherapy (NSAID or acetaminophen) } \\
\text { (reference: no) }\end{array}$ & 0.394 & 1.482 & 1.271 & & 0.310 & 0.757 \\
\hline & \% Specialty pain pharmacotherapy (TCA) (reference: no) & 2.051 & 7.779 & 2.153 & & 0.953 & 0.343 \\
\hline & \% Specialty pain pharmacotherapy (SNRI) (reference: no) & 0.947 & 2.579 & 2.204 & & 0.430 & 0.668 \\
\hline & $\%$ Specialty pain pharmacotherapy (anticonvulsants) (reference: no) & 0.456 & 1.578 & 1.259 & & 0.362 & 0.718 \\
\hline \multirow[t]{5}{*}{ SUD $^{\mathrm{i}}$} & 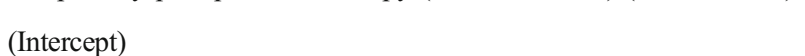 & $\begin{array}{l}\text { Estimate } \\
-4.884\end{array}$ & OR & $\begin{array}{l}\text { SE } \\
0.864\end{array}$ & $\begin{array}{l}\text { DF } \\
47,474\end{array}$ & $\begin{array}{l}\mathrm{t} \text { value } \\
-5.653\end{array}$ & $\begin{array}{l}\mathrm{p} \text { value } \\
<0.001\end{array}$ \\
\hline & Demographic factors & & & & & & \\
\hline & Age (reference: $\leq 30$ ) & -0.269 & 0.764 & 0.032 & 47,474 & -8.316 & $<0.001$ \\
\hline & Gender (reference: female) & 0.035 & 1.036 & 0.077 & & 0.457 & 0.648 \\
\hline & Currently married (reference: no) & -0.091 & 0.913 & 0.041 & & -2.233 & 0.026 \\
\hline \multirow[t]{25}{*}{$\operatorname{SUD}^{\mathrm{i}}$} & & Estimate & OR & $\mathrm{SE}$ & DF & $\mathrm{t}$ value & $\mathrm{p}$ value \\
\hline & $\begin{array}{l}\text { Physical and mental health factors } \\
\text { Medical frailty (reference: no) }\end{array}$ & & 1249 & & \multirow{5}{*}{47,474} & & \\
\hline & $\begin{array}{l}\text { Medical frailty (reference: no) } \\
\text { Drug use disorder (reference: no) }\end{array}$ & $\begin{array}{l}0.222 \\
0.904\end{array}$ & $\begin{array}{l}1.249 \\
2.470\end{array}$ & $\begin{array}{l}0.039 \\
0.049\end{array}$ & & 18.298 & $<0.001$ \\
\hline & Alcohol use disorder (reference: no) & 0.594 & 1.811 & 0.047 & & 12.519 & $<0.001$ \\
\hline & Any mood disorder (reference: no) & 1.679 & 5.362 & 0.079 & & 21.312 & $<0.001$ \\
\hline & Traumatic brain injury (reference: no) & 0.258 & 1.295 & 0.086 & & 2.999 & 0.003 \\
\hline & $\begin{array}{l}\text { Individual-level receipt of clinical practice guideline interventions } \\
\text { SUD specialty treatment or SUD-specific pharmacotherapies } \\
\text { (reference: no) }\end{array}$ & 0.410 & 1.507 & 0.052 & \multirow[t]{9}{*}{47,474} & 7.867 & $<0.001$ \\
\hline & Sedative co-prescription (reference: no) & 0.208 & 1.232 & 0.038 & & 5.430 & $<0.001$ \\
\hline & Regular drug screening (reference: no) & 0.685 & 1.984 & 0.042 & & 16.303 & $<0.001$ \\
\hline & Medication management (reference: no) & 0.373 & 1.451 & 0.040 & & 9.346 & $<0.001$ \\
\hline & Rehabilitation medicine (reference: no) & 0.233 & 1.262 & 0.063 & & 3.724 & $<0.001$ \\
\hline & $\begin{array}{l}\text { Anti-inflammatory pharmacotherapy (NSAID or acetaminophen) } \\
\text { (reference: no) }\end{array}$ & 0.046 & 1.047 & 0.037 & & 1.231 & 0.218 \\
\hline & Specialty pain pharmacotherapy (TCA) (reference: no) & 0.090 & 1.094 & 0.043 & & 2.099 & 0.036 \\
\hline & Specialty pain pharmacotherapy (SNRI) (reference: no) & 0.076 & 1.079 & 0.041 & & 1.850 & 0.064 \\
\hline & Specialty pain pharmacotherapy (anticonvulsants) (reference: no) & 0.247 & 1.281 & 0.037 & & 6.649 & $<0.001$ \\
\hline & $\begin{array}{l}\text { Facility-level receipt of clinical practice guideline interventions } \\
\text { \% SUD specialty treatment or SUD-specific pharmacotherapies } \\
\text { (reference: no) }\end{array}$ & -1.231 & 0.292 & 0.493 & \multirow[t]{10}{*}{128} & -2.495 & 0.014 \\
\hline & $\%$ Sedative co-prescription (reference: no) & -0.292 & 0.747 & 1.854 & & -0.158 & 0.875 \\
\hline & \% Follow-up (reference: no) & -1.111 & 0.329 & 0.702 & & -1.583 & 0.116 \\
\hline & \% Regular drug screening (reference: no) & -1.125 & 0.325 & 0.309 & & -3.645 & $<0.001$ \\
\hline & $\%$ Medication management (reference: no) & 0.454 & 1.574 & 0.401 & & 1.131 & 0.260 \\
\hline & $\%$ Rehabilitation medicine (reference: no) & -0.785 & 0.456 & 0.478 & & -1.641 & 0.103 \\
\hline & $\begin{array}{l}\text { \% Anti-inflammatory pharmacotherapy (NSAID or acetaminophen) } \\
\text { (reference: no) }\end{array}$ & 0.483 & 1.621 & 1.324 & & 0.365 & 0.716 \\
\hline & \% Specialty pain pharmacotherapy (TCA) (reference: no) & 2.344 & 10.426 & 2.205 & & 1.063 & 0.290 \\
\hline & \% Specialty pain pharmacotherapy (SNRI) (reference: no) & 1.206 & 3.339 & 2.241 & & 0.538 & 0.592 \\
\hline & \% Specialty pain pharmacotherapy (anticonvulsants) (reference: no) & 1.398 & 4.048 & 1.295 & & 1.080 & 0.282 \\
\hline
\end{tabular}

\footnotetext{
${ }^{h}$ Overall patient population prescribed CSA opioids

${ }^{i}$ Patient population diagnosed with SUD and prescribed CSA opioids
}

the most anxious and depressed patients, our findings at the patient and facility levels provide the guideline recommendation that sedatives not be prescribed in combination with opioids, at least for patients on LA opioids. 
Table 5. Multivariate Mixed-Effects Logistic Regression Model Predicting Suicide Attempts of the LA Opioid Prescribed Population in the VHA

\begin{tabular}{|c|c|c|c|c|c|c|c|}
\hline \multirow[t]{29}{*}{ Overall } & (Intercept) & $\begin{array}{l}\text { Estimate } \\
-7.289\end{array}$ & OR & $\begin{array}{l}\text { SE } \\
0.870\end{array}$ & $\begin{array}{l}\text { DF } \\
93,367\end{array}$ & $\begin{array}{l}\mathrm{t} \text { value } \\
-8.377\end{array}$ & $\begin{array}{l}\mathrm{p} \text { value } \\
<0.001\end{array}$ \\
\hline & $\begin{array}{l}\text { Demographic factors } \\
\text { Age (reference: }<30 \text { ) }\end{array}$ & -0.266 & 0.766 & 0.044 & \multirow{4}{*}{93,367} & -6.099 & $<0.001$ \\
\hline & Gender (reference: female) & 0.032 & 1.032 & 0.092 & & 0.348 & 0.728 \\
\hline & Currently married (reference: no) & -0.167 & 0.846 & 0.051 & & -3.252 & 0.001 \\
\hline & $\begin{array}{l}\text { Physical and mental health factors } \\
\text { Medical frailty (reference: no) }\end{array}$ & & & & & & \\
\hline & $\begin{array}{l}\text { Medical frailty (reference: no) } \\
\text { Drug use disorder (reference: no) }\end{array}$ & $\begin{array}{l}0.160 \\
1.041\end{array}$ & $\begin{array}{l}1.173 \\
2.832\end{array}$ & $\begin{array}{l}0.052 \\
0.057\end{array}$ & \multirow{5}{*}{93,367} & $\begin{array}{r}3.067 \\
18.276\end{array}$ & $\begin{array}{r}0.002 \\
<0.001\end{array}$ \\
\hline & Alcohol use disorder (reference: no) & 0.627 & 1.871 & 0.056 & & 11.147 & $<0.001$ \\
\hline & Any mood disorder (reference: no) & 1.352 & 3.867 & 0.086 & & 15.664 & $<0.001$ \\
\hline & Traumatic brain injury (reference: no) & 0.408 & 1.504 & 0.147 & & 2.772 & 0.006 \\
\hline & Individual-level receipt of clinical practice guideline interventions & & & & & & \\
\hline & Psychosocial assessment or therapy (reference: no) & 0.896 & 2.450 & 0.082 & \multirow[t]{9}{*}{93,367} & 10.884 & $<0.001$ \\
\hline & Sedative co-prescription (reference: no) & 0.186 & 1.205 & 0.049 & & 3.790 & $<0.001$ \\
\hline & Any drug screening (reference: no) & 0.826 & 2.284 & 0.059 & & 13.893 & $<0.001$ \\
\hline & Medication management (reference: no) & 0.474 & 1.606 & 0.053 & & 8.864 & $<0.001$ \\
\hline & Rehabilitation medicine (reference: no) & 0.268 & 1.307 & 0.083 & & 3.216 & 0.001 \\
\hline & $\begin{array}{l}\text { Anti-inflammatory pharmacotherapy (NSAID or acetaminophen) } \\
\text { (reference: no) }\end{array}$ & 0.040 & 1.041 & 0.050 & & 0.804 & 0.421 \\
\hline & Specialty pain pharmacotherapy (TCA) (reference: no) & 0.054 & 1.055 & 0.055 & & 0.980 & 0.327 \\
\hline & Specialty pain pharmacotherapy (SNRI) (reference: no) & 0.140 & 1.150 & 0.052 & & 2.679 & 0.007 \\
\hline & Specialty pain pharmacotherapy (anticonvulsants) (reference: no) & 0.180 & 1.198 & 0.050 & & 3.596 & $<0.001$ \\
\hline & $\begin{array}{l}\text { Facility-level receipt of clinical practice guideline interventions } \\
\% \text { Psychosocial assessment or therapy (reference: no) }\end{array}$ & -0.625 & 0.535 & 0.406 & \multirow{10}{*}{128} & -1.538 & 0.127 \\
\hline & $\%$ Sedative co-prescription (reference: no) & 3.012 & 20.326 & 1.497 & & 2.012 & 0.046 \\
\hline & $\%$ Follow-up (reference: no) & -1.689 & 0.185 & 0.668 & & -2.528 & 0.013 \\
\hline & $\%$ Any drug screening (reference: no) & -1.086 & 0.338 & 0.295 & & -3.678 & $<0.001$ \\
\hline & $\%$ Medication management (reference: no) & -0.471 & 0.625 & 0.356 & & -1.324 & 0.188 \\
\hline & $\%$ Rehabilitation medicine (reference: no) & -0.294 & 0.745 & 0.496 & & -0.593 & 0.554 \\
\hline & $\begin{array}{l}\text { \% Anti-inflammatory pharmacotherapy (NSAID or acetaminophen) } \\
\text { (reference: no) }\end{array}$ & 0.267 & 1.306 & 1.127 & & 0.237 & 0.813 \\
\hline & \% Specialty pain pharmacotherapy (TCA) (reference: no) & -0.685 & 0.504 & 1.725 & & -0.397 & 0.692 \\
\hline & \% Specialty pain pharmacotherapy (SNRI) (reference: no) & 2.837 & 17.062 & 1.539 & & 1.843 & 0.068 \\
\hline & $\%$ Specialty pain pharmacotherapy (anticonvulsants) (reference: no) & 1.853 & 6.380 & 1.099 & & 1.686 & 0.094 \\
\hline \multirow[t]{5}{*}{$\mathrm{SUD}^{\mathrm{k}}$} & ${ }^{2}$ & $\begin{array}{l}\text { Estimate } \\
-7.284\end{array}$ & OR & SE & DF & $\begin{array}{l}\mathrm{t} \text { value } \\
-7083\end{array}$ & $\begin{array}{l}\mathrm{p} \text { value } \\
<0.001\end{array}$ \\
\hline & Demographic factors & & & & & & \\
\hline & Age (reference: $\leq 30$ ) & -0.221 & 0.802 & 0.055 & 13,546 & -3.991 & $<0.001$ \\
\hline & Gender (reference: female) & 0.131 & 1.140 & 0.136 & & 0.963 & 0.336 \\
\hline & Currently married (reference: no) & -0.073 & 0.930 & 0.067 & & -1.085 & 0.278 \\
\hline \multirow[t]{25}{*}{$\mathrm{SUD}^{\mathrm{k}}$} & - & Estimate & OR & $\mathrm{SE}$ & DF & t value & $\mathrm{p}$ value \\
\hline & Medical frailty (reference: no) & 0.239 & 1.270 & 0.067 & \multirow{5}{*}{13,546} & 3.581 & $<0.001$ \\
\hline & Drug use disorder (reference: no) & 1.090 & 2.974 & 0.094 & & 11.541 & $<0.001$ \\
\hline & Alcohol use disorder (reference: no) & 0.606 & 1.833 & 0.074 & & 8.234 & $<0.001$ \\
\hline & Any mood disorder (reference: no) & 1.775 & 5.899 & 0.144 & & 12.296 & $<0.001$ \\
\hline & Traumatic brain injury (reference: no) & 0.404 & 1.498 & 0.147 & & 2.752 & 0.006 \\
\hline & $\begin{array}{l}\text { Individual-level receipt of clinical practice guideline interventions } \\
\text { SUD specialty treatment or SUD-specific pharmacotherapies (reference: no) }\end{array}$ & 0.317 & 1.373 & 0.083 & \multirow{9}{*}{13,546} & 3.839 & $<0.001$ \\
\hline & Sedative co-prescription (reference: no) & 0.223 & 1.250 & 0.063 & & 3.549 & $<0.001$ \\
\hline & Regular drug screening (reference: no) & 0.430 & 1.537 & 0.071 & & 6.018 & $<0.001$ \\
\hline & Medication management (reference: no) & 0.515 & 1.674 & 0.069 & & 7.519 & $<0.001$ \\
\hline & Rehabilitation medicine (reference: no) & 0.389 & 1.476 & 0.116 & & 3.363 & 0.001 \\
\hline & $\begin{array}{l}\text { Anti-inflammatory pharmacotherapy (NSAID or acetaminophen) } \\
\text { (reference: no) }\end{array}$ & 0.098 & 1.102 & 0.063 & & 1.538 & 0.124 \\
\hline & Specialty pain pharmacotherapy (TCA) (reference: no) & 0.003 & 1.003 & 0.069 & & 0.042 & 0.967 \\
\hline & Specialty pain pharmacotherapy (SNRI) (reference: no) & 0.141 & 1.151 & 0.067 & & 2.099 & 0.036 \\
\hline & $\begin{array}{l}\text { Specialty pain pharmacotherapy (anticonvulsants) (reference: no) } \\
\text { Facility-level receint of clinical practice quideline interventions }\end{array}$ & 0.169 & 1.184 & 0.064 & & 2.635 & 0.008 \\
\hline & $\begin{array}{l}\text { \% SUD specialty treatment or SUD-specific pharmacotherapies } \\
\text { (reference: no) }\end{array}$ & -0.395 & 0.673 & 0.443 & \multirow[t]{10}{*}{128} & -0.892 & 0.374 \\
\hline & \% Sedative co-prescription (reference: no) & 3.409 & 30.235 & 1.690 & & 2.018 & 0.046 \\
\hline & \% Follow-up (reference: no) & -0.440 & 0.644 & 0.766 & & -0.575 & 0.566 \\
\hline & $\%$ Regular drug screening (reference: no) & -0.481 & 0.618 & 0.345 & & -1.393 & 0.166 \\
\hline & $\%$ Medication management (reference: no) & -0.560 & 0.571 & 0.401 & & -1.396 & 0.165 \\
\hline & $\%$ Rehabilitation medicine (reference: no) & -1.156 & 0.315 & 0.537 & & -2.152 & 0.033 \\
\hline & $\begin{array}{l}\text { \% Anti-inflammatory pharmacotherapy (NSAID or acetaminophen) } \\
\text { (reference: no) }\end{array}$ & 0.389 & 1.475 & 1.286 & & 0.302 & 0.763 \\
\hline & \% Specialty pain pharmacotherapy (TCA) (reference: no) & -2.453 & 0.086 & 1.909 & & -1.285 & 0.201 \\
\hline & \% Specialty pain pharmacotherapy (SNRI) (reference: no) & 4.130 & 62.165 & 1.708 & & 2.417 & 0.017 \\
\hline & \% Specialty pain pharmacotherapy (anticonvulsants) (reference: no) & 2.036 & 7.659 & 1.220 & & 1.669 & 0.098 \\
\hline
\end{tabular}

\footnotetext{
${ }^{j}$ Overall patient population prescribed LA opioids

${ }^{k}$ SUD patient population prescribed LA opioids
}

Studies within and outside VA have suggested that patients diagnosed with SUD and other mental health disorders are more likely to receive opioids than patients without these conditions, and that prescription rates have increased more 
rapidly in these higher-risk populations. ${ }^{28}$ VA patients with SUD on chronic OT have also been found to be more likely to show aberrant behaviors that increase drug supply, such as borrowing medication and requesting early refill ${ }^{29}$; these behaviors may additionally increase risk of impulsive intentional overdose. The guideline suggests that SUD diagnosis should be an absolute contraindication for chronic OT unless the patient is receiving SUD treatment. Our findings support that recommendation: we found that (1) SUD-diagnosed patients have substantially higher rates of suicide attempt overall, and (2) among SUD-diagnosed patients prescribed CSA opioids, more consistent receipt of SUD treatment was associated with reduced suicide risk. If treatment is causally related to risk, universal SUD treatment in this population is estimated to reduce suicide attempt risk by a factor of roughly 3.4.

Encouragingly, SUD-diagnosed patients prescribed LA opioids whose care facilities consistently engaged them in rehabilitative treatments that focus on functional improvement had reduced risk of suicide attempt. These rehabilitative treatments may not only be effective for reducing pain, but may also improve function and decrease disability. Disability may contribute to suicidality ${ }^{30,31}$; rehabilitative treatments may limit suicide attempts by reducing functional impairment. The finding that SUD-diagnosed patients prescribed LA opioids have substantially elevated suicide attempt risk at facilities where SNRIs are prescribed at higher rates was unexpected, and should be explored in future studies before drawing inferences.

Our analysis has several limitations. First, this study examines associations in cross-sectional data; thus causality and directionality of identified relationships cannot be determined. Additionally, this study relies on administrative coding of diagnoses and adverse events in the electronic medical record. The accuracy and consistency of this coding may vary by provider. Moreover, VA may not be notified of all suicide attempts or deaths. In 2008, however, the VA Uniform Mental Health Services Handbook ${ }^{32}$ instituted a requirement that all VA facilities employ suicide prevention coordinators, whose role includes reliably documenting and flagging patients with suicide attempts in the medical record to facilitate proper management of these patients. We expect that the availability of these staff at each VA facility improved reliability of suicide attempt coding across VA facilities, though it is likely that there is still some under-counting of events. Additionally, this study looks only at use of practices from the $C P G$ for $O T$ and does not comprehensively consider health care practices that might reduce suicide risk for this population. Lastly, we did not have data regarding the severity of Veteran patient pain intensity, functional limitations, chronicity and response to pain treatments. These factors have associated with suicide risk $^{33}$ and may underlie some of the associations observed in this study.

The associations identified between use of guideline recommendations for OT and risk of suicide attempt provide a rational basis for prioritizing implementation of guideline recommendations and encouraging facility-level adherence to the prioritized guideline recommendations. Future trials are needed to determine whether implementation of guideline recommendations for OT can reduce risk of suicide attempt for high risk populations. The findings here should help guide development and targeting of implementation interventions for such a trial.

Acknowledgements: This project was funded by grant RRP 10-106 entitled "Assessment of Gaps in Chronic Opioid Therapy Guideline Adherence" from the VA Substance Use Disorders Quality Enhancement Research Initiative. The authors gratefully acknowledge Dr. Margaret Brandeau for her editorial comments. The views expressed are those of the authors and do not necessarily reflect positions or policies of the Department of Veterans Affairs or of the United States government.

Conflict of Interest: The authors have no conflict of interest to report.

Corresponding Author: Jinwoo J. Im, PhD; Management of Innovation ProgramDaegu Gyeongbuk Institute of Science and Technology, 333 Techno jungang-daero, Hyeonpung-myeon, Dalseong-gun, Daegu 711-873, South Korea (e-mail: jinwooim@gmail.com).

\section{REFERENCES}

1. Tang NK, Crane C. Suicidality in chronic pain: a review of the prevalence, risk factors and psychological links. Psychol Med. 2006;36:575-86.

2. Ilgen MA, Zivin K, Austin KL, Bohnert AS, Czyz EK, Valenstein M, Kilbourne AM. Severe pain predicts greater likelihood of subsequent suicide. Suicide Life Threat Behav. 2010;40:597-608.

3. Breslau N, Schultz L, Lipton R, Peterson E, Welch KM. Migraine headaches and suicide attempt. Headache. 2012;52:723-31.

4. Braden JB, Sullivan MD. Suicidal thoughts and behavior among adults with self-reported pain conditions in the national comorbidity survey replication. J Pain. 2008;9:1106-15.

5. Hakansson AF, Schlyter F, Berglund M. Factors associated with history of non-fatal overdose among opioid users in the Swedish criminal justice system. Drug Alcohol Depend. 2008;94:48-55.

6. Ilgen MA, Zivin K, McCammon RJ, Valenstein M. Pain and suicidal thoughts, plans and attempts in the United States. Gen Hosp Psychiatry. 2008;30:521-7.

7. Magni GS, Rigatti-Luchini S, Fracca F, Merskey H. Suicidality in chronic abdominal pain: an analysis of the Hispanic Health and Nutrition Examination Survey (HHANES). Pain. 1998;76:137-44.

8. Ratcliffe GE, Enns MW, Belik SL, Sareen J. Chronic pain conditions and suicidal ideation and suicide attempts: an epidemiologic perspective. Clin J Pain. 2008;24:204-10.

9. Theodoulou ML, Harriss $\mathbf{L}$, Hawton $\mathbf{K}$, Bass $\mathbf{C}$. Pain and deliberate selfharm: an important association. J Psychosom Res. 2005;58:317-20.

10. Cheatle MD. Depression, chronic pain, and suicide by overdose: on the edge. Pain Med. 2011;12:S43-S48.

11. VA/DoD Opioid Therapy for Chronic Pain Working Group. Clinical practice guideline: management of opioid therapy for chronic pain (2010). Available at: http://www.healthquality.va.gov/Chronic_Opioid_Therapy_COT.asp. Accessed on January 20, 2015.

12. Chou R, Fanciullo GJ, Fine PG, Adler JA, Ballantyne JC, Davies P, Donovan MI, Fishbain DA, Foley KM, Fudin J, Gilson AM, Kelter A Mauskop A, O'Connor PG, Passik SD, Pasternak GW, Portenoy RK, Rich BA, Roberts RG, Todd KH, Miaskowski C. American pain societyAmerican academy of pain medicine opioids guidelines panel. Clinical guidelines for the use of chronic opioid therapy in chronic noncancer pain. Clin J Pain. 2009; 10:113-30.

13. Kalso E, Allan L, Dellemijn PLI, Faura CC, Ilias WK, Jensen TS, Perrot S, Plaghki LH, Zenz M. Recommendations for using opioids in chronic non-cancer pain. Eur J Pain. 2003;7:381-386.

14. Midboe AM, Lewis ET, Paik MC, et al. Measurement of adherence to clinical practice guidelines for opioid therapy for chronic pain. Transl Behav Med. 2012;2:57-64. 
15. Finney JW, Humphreys K, Kivlahan DR, Harris AH. Why health care process performance measures can have different relationships to outcomes for patients and hospitals: understanding the ecological fallacy. Am J Public Health. 2011;101:1635-42.

16. Buscaglia AC, Paik MC, Lewis E, Trafton JA, VA Opioid Metric Development Team. Baseline variation in use of VA/DOD clinical practice guideline recommended opioid prescribing practices across VA health care systems. Clin J Pain. in press.

17. Li Y. Power Analysis for a Mixed Effects Logistic Regression Model. PhD Dissertation. Louisiana State University, Baton Rouge, 2006.

18. Venables WN, Ripley BD. Modern applied statistics with S. 4th ed. New York: Springer; 2002. ISBN:0-387-95457-0.

19. R Development Core Team (2012). R: A language and environment for statistical computing. R Foundation for Statistical Computing, Vienna, Austria. ISBN 3-900051-07-0, URL http://www.R-project.org/.

20. Vijayakumar L, Kumar MS, Vijayakumar V. Substance use and suicide. Curr Opin Psychiatry. 2011;24(3):197-202.

21. Mann JJ, Apter A, Bertolote J, Beautrais A, et al. Suicide prevention strategies: a systematic review. JAMA. 2005;294(16):2064-74.

22. Toblin RL, Paulozzi LJ, Logan JE, Hall AJ, Kaplan JA. Mental illness and psychotropic drug use among prescription drug overdose deaths: a medical examiner chart review. J Clin Psychiatry. 2010;71:491-6.

23. Häkkinen M, Launiainen T, Vuori E, Ojanperä I. Comparison of fatal poisonings by prescription opioids. Forensic Sci Int. 2012;222:327-31.

24. Paulozzi LJ, Kilbourne EM, Shah NG, Nolte KB, Desai HA, Landen MG, Harvey W, Loring LD. A history of being prescribed controlled substances and risk of drug overdose death. Pain Med. 2012;13:8795.
25. Brower KJ, McCammon RJ, Wojnar M, Ilgen MA, Wojnar J, Valenstein M. Prescription sleeping pills, insomnia, and suicidality in the national comorbidity survey replication. J Clin Psychiatry. 2011;72:515-21.

26. Kobus AM, Smith DH, Morasco BJ, Johnson ES, Yang X, Petrik AF, Deyo RA. Correlates of higher-dose opioid medication use for low back pain in primary care. Clin J Pain. 2012;13:1131-8.

27. Seal KH, Shi Y, Cohen G, Cohen BE, Maguen S, Krebs EE, Neylan TC. Association of mental health disorders with prescription opioids and highrisk opioid use in US veterans of Iraq and Afghanistan. JAMA. 2012;307:940-7.

28. Edlund MJ, Martin BC, Devries A, Fan MY, Braden JB, Sullivan MD. Trends in use of opioids for chronic noncancer pain among individuals with mental health and substance use disorders: the TROUP study. Clin J Pain. 2010;26:1-8.

29. Morasco BJ, Dobscha SK. Prescription medication misuse and substance use disorder in VA primary care patients with chronic pain. Gen Hosp Psychiatry. 2008;30:93-9.

30. Fisher BJ, Haythornthwaite JA, Heinberg LJ, Clark M, Reed J. Suicidal intent in patients with chronic pain. Pain. 2001;89:199-206.

31. Pirkis J, Burgess P, Dunt D. Suicidal ideation and suicide attempts among Australian adults. Crisis. 2000;21:16-25.

32. Veterans Health Administration, Department of Veterans Affairs. Uniform mental health services in VA medical centers and clinics (2008). Available at: http://wwwl.va.gov/vhapublications/ViewPublication.asp?pub_ ID=1762. Accessed on January 20, 2015.

33. Tang NK, Crane C. Suicidality in chronic pain: a review of the prevalence, risk factors and psychological links. Psychol Med. 2006;36(5):575-86

\section{APPENDICES}

\section{Appendix 1}

Table 6. Internal Entry Numbers (IENs)* Used to Identify and Categorize Opioids

\begin{tabular}{lll}
\hline \hline Type of opioids & & IENs \\
\hline Short-acting & Codeine & $00027-00029,00036,00039,00040,00679-00681,01838-01847,04300,05061,05062,05240-05242$, \\
& & $05919-05923,06099-06103,07105-07107,07119,07120,07180,07181,07239,13911,15762$ \\
& Morphine & $00355-00357,00363-00367,00372-00374,00376,00377,00379,00381,00383,00385,00386,15713$, \\
& Propoxyphene & $00594-00597,00675-00678,01774-01778,07995,07996,15130,16563,17324$ \\
& Levorphanol & 01587 \\
& Oxycodone & $02482-02488,02490,04602-04604,04608,04610,14291,14293,14294,14740,15611,16537$, \\
& Meperidine & $02639,02643,02646,02647,02658,03691,15709$ \\
& Hydromorphone & $02695-02699,02705,02710,15710$ \\
& Dihydrocodeine & $02821,02835,06612,06912,07244,15610,21187$ \\
& Butorphanol & 03270 \\
& Pentazocine & $03789,03791,03792$ \\
& Fentanyl & $05804-05806,13981-13984,18015-18018$ \\
& Hydrocodone & $04410-04423,06421,07203,07610,07611,07666,12782,13360-13362,15046,15915,16373$, \\
& Oxymorphone & $07147,17696,19984,20205$ \\
& Tapentadol & $20923-20925$ \\
Methadone & $00135,00136,00138-00166,15703,20101$ \\
Long-acting & $00362,00378,00387,00394-00396,15361,15362,15712,15714,15763,16556,16557$, \\
& Morphine & $20983-20986,20988,20989,21479$ \\
& Oxycodone & $04605-04607,04609,14617,15716-15718,19163-19165$ \\
& Fentanyl & $05799-05802,17264,17697$ \\
& Hydromorphone & $17319-17322$ \\
& Oxymorphone & $17833-17840,20834,21341$ \\
\hline
\end{tabular}

* The first five digits of the VHA Decision Support System (DSS) feeder key. We used this number to find pharmacy records in the DSS Pharmacy Laboratory National Data Extracts (NDEs). The DSS feeder key contains a 17-digit number that identifies the drug or supply dispensed. The first five digits contain an IEN that points to the entry in the VistA VA PRODUCT File (\#50.68) for the drug dispensed. The last 12 digits contain the 12-digit version of the National Drug Code (NDC). The 17-digit number may be used to link the records to the National Drug File (NDF)

(Source: http://www.virec.research.va.gov/RUGs/RUG-PBM-2nd-Ed-CY08-ER.pdf) 


\section{Appendix 2}

Table 7. International Classification of Diseases, Ninth Revision,

Clinical Modification (ICD-9-CM) Codes Used to Identify Suicides or Suicide Attempts

\begin{tabular}{ll}
\hline \hline & ICD-9-CM codes \\
\hline $\begin{array}{l}\text { Possible and confirmed } \\
\text { suicide attempts }\end{array}$ & E950-E959, E980.6, E980.8, \\
\hline
\end{tabular}

\section{Appendix 3}

Table 8. International Classification of Diseases, ninth revision, Clinical Modification (ICD-9-CM) Codes Used to Identify Physical and Mental Health Status

\begin{tabular}{|c|c|c|}
\hline & & ICD-9-CM codes \\
\hline $\begin{array}{l}\text { Drug use disorder } \\
\text { Alcohol use disisorc } \\
\text { Mood disorder }\end{array}$ & $\begin{array}{l}\text { Peptic ulcer disease - excluding bleeding } \\
\text { AIDS } \\
\text { Lymphoma } \\
\text { Metastatic cancer } \\
\text { Solid tumor without metastasis } \\
\text { Rheumatoid arthritis/collagen vascular } \\
\text { Coagulopathy } \\
\text { Weight loss } \\
\text { Fluid and electrolyte disorders } \\
\text { Blood loss anemia } \\
\text { Deficiency anemias } \\
\text { ler } \\
\text { Depression } \\
\text { Anxiety disorder } \\
\text { PTSD } \\
\text { Affective psychosis (bipolar) } \\
\text { jury }\end{array}$ & $\begin{array}{l}398.91,402.11,402.91,404.11,404.13,404.91,404.93,428 \\
426.10,426.11,426.13,427.0,427.2,427.31,427.60,427.9,785.0, \mathrm{~V} 45.0, \mathrm{~V} 53.3, \\
426.2-426.53,426.6-426.89 \\
\text { V42.2,V43.3, 093.20-093.24, 394.0-397.1, 424.0-424.91, 746.3-746.6 } \\
416,417.9 \\
441.2,441.4,441.7,441.9,447.1,557.1,557.9, \mathrm{~V} 43.4,440.0-440.9,443.1-443.9 \\
342.0-342.12,342.9-344.9 \\
331.9,332.0,333.4,333.5,340,348.1,348.3,780.3,784.3,334.0-335.9,341.1-341.9, \\
345.00-345.11,345.40-345.51,345.80-345.91 \\
494,506.4,490-492.8,493.00-493.91,495.0-505 \\
243-244.2,244.8-244.9 \\
403.11,403.91,404.12,404.92,585,586, \mathrm{~V} 42.0, \mathrm{~V} 45.1, \mathrm{~V} 56.0, \mathrm{~V} 56.8 \\
070.32,070.33,070.54,456.0,456.1,456.20,456.21,571.0,571.2,571.3, \\
571.5,571.6,571.8,571.9,572.3,572.8, \mathrm{~V} 42.7,571.40-571.49 \\
531.70,531.90,532.70,532.90,533.70,533.90,534.70,534.90, \mathrm{~V} 12.71 \\
042 \\
238.6,273.3, \mathrm{~V} 10.7,200.00-202.38,202.50-203.01,203.8-203.81 \\
196,197,198,199.0,199.1 \\
174,175, \mathrm{~V} 10,140.0-172.9,179-195.8 \\
701.0,710,714,720,725 \\
286,287.1,287.3,287.4,287.5 \\
260,261,262,263 \\
276 \\
280.0 \\
285.9 \\
292,304,305.2-306 \\
291,303,305.0 \\
311,309.0,309.1,301.12,300.4 \\
300.02 \\
309.81 \\
296.0-296.2,296.4-296.9 \\
850.0-859.9\end{array}$ \\
\hline
\end{tabular}

* Patients with any of the codes in the right-hand column were coded to have medical frailty

+ Patients with any of the codes in the right-hand column were coded to have mood disorder 


\section{Appendix 4}

Table 9. Coding Definitions Used to Identify and Categorize Clinical Practice Guideline (CPG)-Based Services

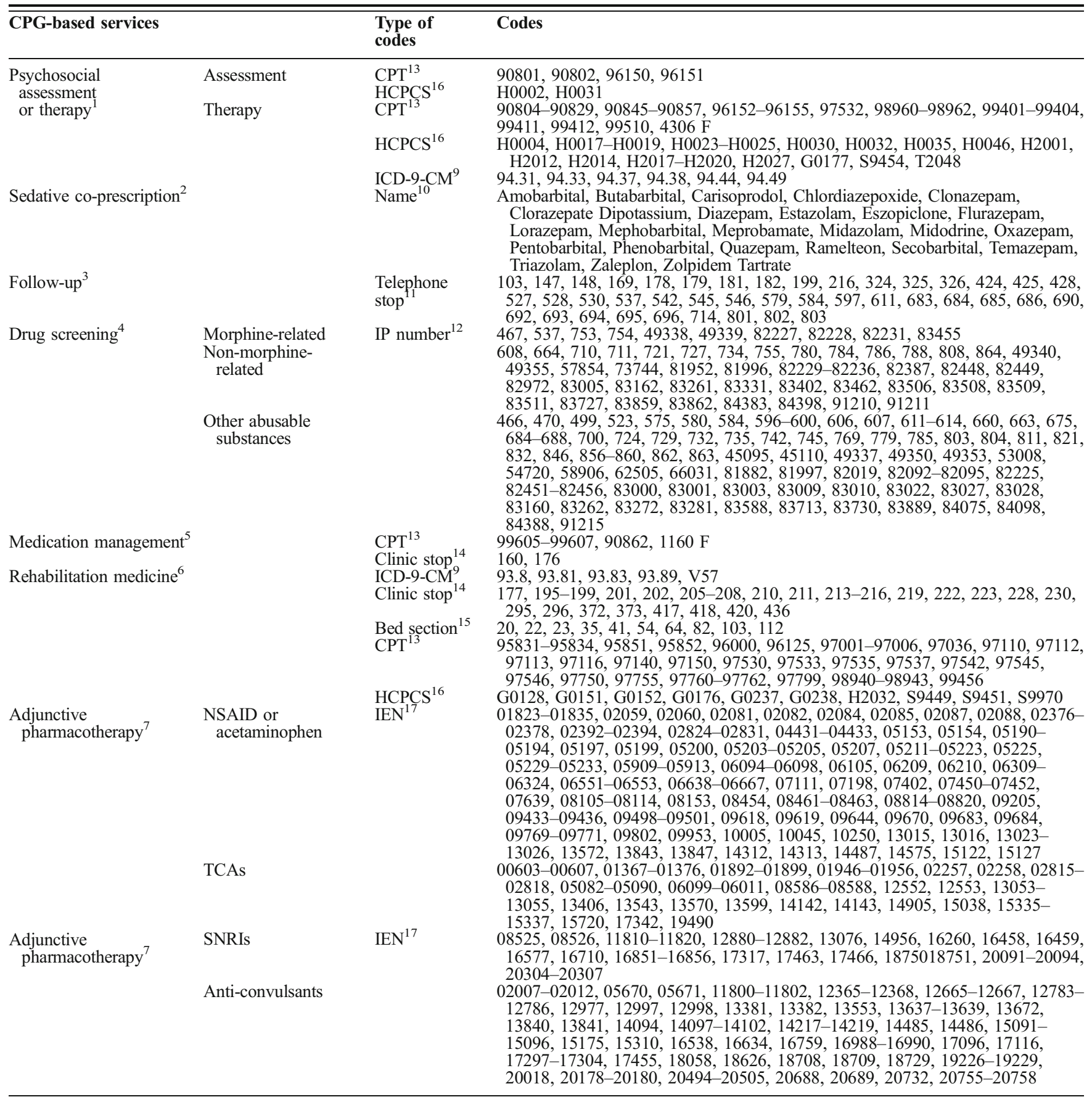


Table 9. (continued)

\begin{tabular}{|c|c|c|}
\hline CPG-based services & $\begin{array}{l}\text { Type of } \\
\text { codes }\end{array}$ & Codes \\
\hline \multirow[t]{3}{*}{$\begin{array}{l}\text { SUD specialty treatment or } \\
\text { SUD-specific pharmacotherapies }\end{array}$} & ICD-9-CM ${ }^{9}$ & $\begin{array}{l}291-292.9,303.00-303.02,303.90-303.92,304.00-304.02,304.10-304.12, \\
304.20-304.22,304.30-304.32,304.40-304.42,304.50-304.52,304.60-304.62, \\
304.70-304.72,304.80-304.82,304.90-304.92,305.00-305.02,305.20-305.22, \\
305.30-305.32,305.40-305.42,305.50-305.52,305.60-305.62,305.70-305.72, \\
305.80-305.82,305.90-305.92\end{array}$ \\
\hline & Clinic stop ${ }^{14}$ & $\begin{array}{l}292,502-514,516,519,522-525,529,531-535,539,540,547,548,550,552- \\
554,557-562,564,565,567,568,571-577,580,582-584,588,590-596,598, \\
599\end{array}$ \\
\hline & $\begin{array}{l}\text { Bed section } \\
\text { Name }^{10}\end{array}$ & $\begin{array}{l}25-27,37,39,85,88,109-111 \\
\text { Buprenorphine, Subutex, Suboxone, Acamprosate, Disulfiram }\end{array}$ \\
\hline
\end{tabular}

${ }^{1}$ OT patients should receive a full mental health assessment, coping skills and stress management training or psychotherapy procedures within the year ${ }^{2}$ OT patients should not receive overlapping prescriptions for opioids and sedative medications such as barbiturates, benzodiazepines, and carisoprodol (SOMA)

${ }^{3}$ Patients receiving new opioid prescriptions should have a clinical encounter with VHA within 4 weeks. Because this variable is only relevant to patients with new opioid prescriptions, it was only included as a facility-level variable in order to retain all patients in the analyses

${ }^{4}$ Drug screening can identify patients using illicit substances and who may be in need of additional services and/or support. Medical service providers are recommended to order routine and random drug screenings for all patients with chronic pain prior to and during OT. The laboratory drug screens for heroin/morphine, non-morphine opioid compounds, and non-opioid abusable substances were included

${ }_{5}$ OT patients should receive pharmacy reconciliation documented by a pharmacy reconciliation encounter

6,7 Avoidance of sole reliance on opioids, as defined by receipt of: (a) rehabilitation therapies including physical therapy, physical medicine and rehabilitation, recreational therapy, occupational therapy, pain clinic, or a prescribed exercise program; non-opioid pharmacotherapies including (b) anti-inflammatories (NSAID or acetaminophen), (c) tricyclic antidepressants (TCAs), (d) serotonin-norepinephrine reuptake inhibitors (SNRIs), or (e) anticonvulsants. Note that patients may or may not have received these interventions or prescriptions specifically for their pain condition. These measures examine only whether patients received an intervention or prescription that has been suggested to be beneficial for pain conditions per clinical practice guidelines

${ }^{8}$ SUD patients should receive SUD specialty treatment or SUD-specific pharmacotherapies while receiving opioid therapy; in addition, SUD patients should receive regular drug screening, defined as at least one for every 90-day supply of the opioid

${ }^{9}$ ICD-9-CM: International Classification of Diseases, Ninth Revision, Clinical Modification codes

${ }^{10}$ Name: Names of the sedatives are used to search by drug description in the VHA Decision Support System (DSS) Pharmacy Laboratory National Data Extracts (NDEs)

${ }_{11}^{11}$ Telephone stop: Codes used to track follow-ups

${ }^{12}$ IP number: Intermediate Product number found in the DSS Laboratory NDEs. This number is a pointer to the DSS Product Table, which contains information specific to the service provided. The product table includes feeder system, intermediate product department, feeder key, and a description of the procedure

(Source: http://www.virec.research.va.gov/RUGs/RUG-DSS-NDE-2nd-Ed-CY09-ER.pdf)

${ }_{13}$ CPT: Current Procedural Terminology codes used to record procedures in the DSS NDES

${ }_{15}^{14}$ Clinic stop: Codes used to record procedures in outpatient visits

15 Bed section: Codes used in the recording of inpatient stays

${ }^{16}$ HCPCS: Health Care Common Procedure Coding System codes used to record procedures in the DSS NDES

17 IEN: The first five digits of the VHA DSS feeder key used to find pharmacy records in the DSS Pharmacy Laboratory NDEs (Source: http:// www.virec.research.va.gov/RUGs/RUG-PBM-2nd-Ed-CY08-ER.pdf) 